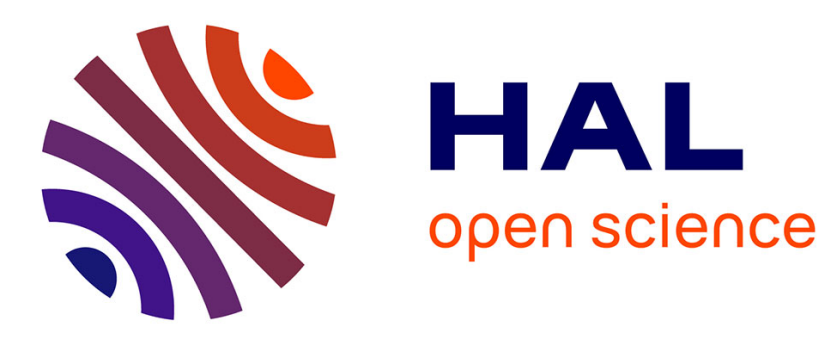

\title{
Hiérarchies socio-professionnelles et violence interpersonnelle dans l'Égypte du Nouvel Empire et d'époque hellénistique
}

Christine Hue-Arcé

\section{- To cite this version:}

Christine Hue-Arcé. Hiérarchies socio-professionnelles et violence interpersonnelle dans l'Égypte du Nouvel Empire et d'époque hellénistique. Archimède : archéologie et histoire ancienne, 2017, 4, pp.174183. halshs-01589363

\section{HAL Id: halshs-01589363 \\ https://shs.hal.science/halshs-01589363}

Submitted on 18 Sep 2017

HAL is a multi-disciplinary open access archive for the deposit and dissemination of scientific research documents, whether they are published or not. The documents may come from teaching and research institutions in France or abroad, or from public or private research centers.
L'archive ouverte pluridisciplinaire HAL, est destinée au dépôt et à la diffusion de documents scientifiques de niveau recherche, publiés ou non, émanant des établissements d'enseignement et de recherche français ou étrangers, des laboratoires publics ou privés. 


\section{ARCHIMÈDE N4}

\section{DOSSIER THÉMATIQUE 1 : NOMMER LES « ORIENTAUX » DANS L’ANTIQUITÉ}

\section{DOSSIER THÉMATIQUE 2 : PRYTANÉE ET REGIA}

\section{ACTUALITÉ DE LA RECHERCHE : DYNAMIQUES HUMAINES ANCIENNES}

155 Steeve GENTNER, Thomas HUTIN et Šárka VÁLEČKOVÁ

Introduction au dossier : les phénomènes de hiérarchisation et leurs traitements dans les sociétés anciennes et actuelles

158 Pierre LE ROUX et Alain BEYRAND

D'un drame à un don. Hommage à Louise Beyrand et Olivier Toussaint

162 Alexandra CONY

Hiérarchie de I'habitat rural à la fin de l'âge du Fer. L'importance de définir les critères de hiérarchisation en fonction de l'environnement archéologique

174 Christine HUE-ARCE

Hiérarchies socio-professionnelles et violence interpersonnelle dans l'Égypte du Nouvel Empire et d'époque hellénistique

184 Aurélien LANDON

La hiérarchie dans le recrutement militaire : I'exemple épigraphique du recrutement des tribuns militaires durant le Principat d'Auguste

196 Sophie TRIERWEILER

La conception et l'expression d'un droit structuré dans la société homérique à travers les notions de themis et dikê

207 François FAVORY

Organisation et hiérarchisation de l'habitat antique : l'expérience d'Archaeomedes et d'ArchaeDyn

VARIA

216 Jean-Claude LACAM

Prestota Serfia Serfer Martier, la déesse immobile (étude ombrienne, $\mathrm{III}^{\mathrm{e}}-\mathrm{II}^{\mathrm{e}}$ siècles av. J.-C.)

229 Pierre SCHNEIDER

Des Indiens dans les armées hellénistiques : une autre rencontre entre la Grèce et I'Inde

\section{LA CHRONIQUE D’ARCHIMÈDE}

236 Frédéric COLIN (éd.)

La Chronique d'Archimède. Bilan des activités scientifiques 2016-2017 de I'unité mixte

\section{@creative}




\section{HIÉRARCHIES SOCIO-PROFESSIONNELLES ET VIOLENCE INTERPERSONNELLE DANS L'ÉGYPTE DU NOUVEL EMPIRE ET D'ÉPOQUE HELLÉNISTIQUE [1]}

\section{Christine HUE-ARCÉ}

Docteure en égyptologie

Université de Strasbourg UMR 7044 Archimède

christine.huearce@gmail.com

\section{RÉSUMÉ}

Cet article analyse les mécanismes de traitement de la violence interpersonnelle exercée dans le cadre d'une hiérarchie sociale ou professionnelle, dans la documentation égyptienne du Nouvel Empire et d'époque hellénistique. L'auteure interroge la perception des violences infligées à un individu en position d'infériorité par son supérieur, et dénoncées dans la documentation : s'agit-il réellement d'actes de violence répréhensibles, ou ces violences sontelles considérées comme légitimes par les auteurs des textes égyptiens ? L'étude de corpus documentaires de deux époques distantes permet

Mots-CLÉS

Conflits,

hiérarchie,

Égypte ancienne,

époque hellénistique,

Nouvel Empire,

violence interpersonnelle. en outre de souligner les convergences de ce phénomène social entre le Nouvel Empire et la période hellénistique.
Through the study of Egyptian documents from New Kingdom and Hellenistic Egypt, this article analyses the mechanisms of the treatment of interpersonal violence occurring in the framework of a social or professional hierarchy. The author questions the actual perception of violence inflicted by a superior to their inferior, and denounced in the texts: was this violence seen as reprehensible, or was it perceived as legitimate by the authors of the Egyptian texts? The study of bodies of documents from two separate periods enables highlighting the common patterns of this social phenomenon between the New Kingdom and the Hellenistic period.
KeYWORDS Ancient Egypt, conflicts, Hellenistic Period, Hierarchy,

New Kingdom, interpersonal Violence. 
« Complex societies and states are

articulated by relations of power. »

FROOD 2010, p. 476.

L'Égypte du Nouvel Empire et d'époque hellénistique n'échappe pas à cette constatation d'Elizabeth Frood. Les relations hiérarchiques participent à un réseau de pouvoir et de soutien, organisé autour des liens de parenté, de subordination, de dépendance et de patronage.

Les relations de pouvoir qui articulent la société égyptienne s'expriment par un soutien au faible, au pauvre ou à l'isolé. Les textes révèlent pourtant que ces relations pouvaient parfois dégénérer, des abus naître, et la violence surgir. Cette étude est consacrée aux actes de violence interpersonnelle qui surviennent dans le cadre d'une hiérarchie sociale ou professionnelle, rapportés dans des textes égyptiens du Nouvel Empire ( $\mathrm{xvI}^{\mathrm{e}}-\mathrm{x}^{\mathrm{e}}$ s. av. J.-C.) et d'époque hellénistique (332-30 av. J.-C.).

La notion de hiérarchie est à entendre ici comme toute « organisation sociale établissant des rapports de subordination et des degrés gradués de pouvoirs, de situation et de responsabilités »[2]. Les hiérarchies professionnelles et sociales seront au centre de I'enquête, englobant non seulement les différences de rang social, mais aussi de niveau de vie (ou, pour le dire de manière plus sommaire, l'opposition entre pauvres et riches). La question de la hiérarchie des genres - et de la place de la femme dans la société égyptienne, sujet vaste et complexe- ne sera pas abordée dans le présent article [3].

Cet article analyse plus spécifiquement les actes de violence physique interpersonnelle, qui surviennent

[1] Cet article est une recherche issue de ma thèse de doctorat, soutenue en septembre 2015 à I'Université de Strasbourg (cf. Hue-Arcé 2015). Je tiens à remercier Sophie Trierweiler pour ses remarques et corrections, ainsi que le « reviewer » anonyme pour ses suggestions enrichissantes. Sauf indication contraire, toutes les traductions de textes égyptiens présentées dans cet article sont des traductions personnelles.

[2] Définition B du Centre National de Ressources Textuelles et Lexicales (CNRTL), « Hiérarchie » [en ligne] http://www.cnrtl.fr/definition/hi\%C3\%A9rarchie [page consultée le 22-02-2016]. dans le cadre des relations sociales, de la vie quotidienne, et sont perçus par l'auteur d'un texte comme répréhensibles, illégitimes [4]. La notion de violence interpersonnelle exclut ainsi I'usage d'une force physique qui semble, du point de vue du lecteur moderne occidental, constituer un acte de violence, mais qui est présenté par l'auteur d'un texte égyptien comme un geste légitime, justifié. La focalisation sur une violence dénoncée, et donc réellement perçue comme telle par son dénonciateur, vise à éviter l'écueil d'une compréhension de la violence basée sur nos conceptions occidentales (étique), et permet de se rapprocher du point de vue interne à la société égyptienne (émique) [5].

Deux principaux ensembles documentaires seront examinés dans cet article : d'une part, les textes de la pratique quotidienne du Nouvel Empire, dont une grande partie est issue de Deir el-Médina, le village des artisans de la nécropole thébaine ; d'autre part les textes de la pratique quotidienne rédigés dans la cursive démotique pour l'époque hellénistique. Des textes littéraires des deux époques, qui apportent un éclairage supplémentaire sur la violence dans un contexte hiérarchique, seront également pris en considération. Deir el-Médina est un village particulier, créé pour et habité par des artisans, ainsi que leurs familles et serviteurs. Les sources issues de ce microcosme [6] doivent être examinées avec précaution : les conclusions qui en sont tirées ne peuvent pas toujours être appliquées au
[3] J'ai abordé ailleurs le thème de la violence exercée à l'encontre des femmes pour l'époque gréco-romaine, cf. Hue-Arcé (à paraître). Pour le Nouvel Empire, consulter entre autres ToIVARI-VIITALA 2001, p. 216-219. Sur la place des femmes dans la société égyptienne, voir notamment DieLEMAN 1998 et JoHNSON 1996.

[4] Cf. Riess 2012, p. 3-4.

[5] Sur les notions d'emic et d'etic, développées en linguistique par I'américain Kenneth Pike, cf. PIKE 1967, p. 37-39.

[6] Pour la qualification, usuelle, de Deir el-Médina de « microcosme », voir par ex. WARD 1994, p. 69. 


\begin{tabular}{|c|c|c|c|c|}
\hline & DOCUMENT & TRANSLITTÉRATION & TRADUCTION & DATATION \\
\hline Louvre E 11673 & $\begin{array}{l}\text { Contrat } \\
\text { de mariage }\end{array}$ & $h w$ & Frapper & $\begin{array}{c}\text { XVIII } \text { dynastie } \\
\text { (an } 27 \text { de Thoutmosis III) }\end{array}$ \\
\hline $\begin{array}{l}\text { O. Mond 237/ } \\
\text { O. ROM 906.19.5 }\end{array}$ & \multirow{3}{*}{ Lettres } & Qnqn & Frapper/Battre & $\begin{array}{c}\text { XIXe dynastie } \\
\text { (an 16-30 de Ramsès II) }\end{array}$ \\
\hline O. Ashm. Mus. 32 & & Sh.t & Coup & Fin de la $X I X^{e}$ ou $X X^{e}$ dynastie \\
\hline P. Genève D 187 & & Qnqn & Frapper/Battre & $\begin{array}{c}\mathrm{XX} \text { dynastie } \\
\text { (Ramsès XI, an début du whm-msw.t) }\end{array}$ \\
\hline \multirow{4}{*}{ P. BM EA 10055} & \multirow{4}{*}{ Plainte } & $\underline{h} d b$ & Tuer & \multirow{4}{*}{ Fin de la XIXe dynastie } \\
\hline & & Qnqn & Frapper/Battre & \\
\hline & & $h w 3 d b . w t$ & Jeter des briques & \\
\hline & & hws $i n r$ & Jeter des pierres & \\
\hline
\end{tabular}

Figure 1 : actes de violence interpersonnelle dans le cadre d'une relation hiérarchique dans les textes documentaires du Nouvel Empire.

macrocosme que représente l'ensemble de l'Égypte. Cependant, pour le sujet qui nous intéresse, certains textes proviennent de Thèbes, et non du village d'artisans : les conclusions concernant la violence interpersonnelle en contexte hiérarchique pour le Nouvel Empire ne concerneront donc pas la seule Deir el-Médina. Pour la période gréco-romaine, le choix de restreindre la présente étude à la seule époque hellénistique est en réalité imposé par la datation des textes : les documents démotiques qui nous renseignent sur cette thématique sont en effet concentrés sur la période ptolémaïque.

L'objectif de cet article est de souligner les caractéristiques du traitement d'agressions, qui semblent de prime abord relever de la catégorie des violences interpersonnelles, lorsqu'elles surviennent dans le cadre d'une relation hiérarchique. Je m'attarderai notamment sur la perception de ces actes : cette violence était-elle véritablement perçue négativement et constituait-elle une réelle violence interpersonnelle ? Dans quel(s) contexte(s) une violence hiérarchique pouvait-elle être considérée comme illégitime ? La comparaison des données de deux époques différentes, pour lesquelles une documentation cohérente est disponible, permet de questionner l'existence de convergences et la continuité des phénomènes sociaux entre les deux périodes. Dans I'optique de cette mise en évidence des caractéristiques communes au Nouvel Empire et à l'époque

[7] LiNAGE 1939, p. 217-234.

[8] $N n h w . t w=f n n \check{s} n\left[{ }^{\prime} . t w=f\right] h r r s b 3 n b n n s w$. Louvre E $11673,10-11$.

[9] Cf. EYRE 1992, p. 215. Il semble en effet probable que Sa-Bastet n'ait pas eu de parent masculin susceptible de lui succéder à la tête de sa maisonnée.

[10] Cf. EYRE 1992, p. 215. hellénistique, une présentation chronologique des textes et de leur interprétation sera privilégiée, pour ensuite confronter ces résultats.

\section{VIOLENCE, HIÉRARCHIE(S) ET PATRONAGE AU NOUVEL EMPIRE}

Quelques textes du Nouvel Empire présentent des actes de violence entre des individus engagés dans une relation hiérarchique. La plupart d'entre eux sont issus de la documentation de la pratique quotidienne : un contrat de mariage et trois lettres; la question du contexte hiérarchique d'actes de violence rapportés dans une plainte sera également posée (fig. 1).

La statuette Louvre E 11673 [7] porte un contrat de mariage, daté du règne de Thoutmosis III, établi par le barbier royal Sa-Bastet à propos de l'union de sa nièce Nébetta et de son ancien serviteur Imeniouy. Dans ce texte, le barbier promet qu'Imeniouy « ne sera pas frappé, il ne sera retenu à aucune porte du roi » [8]. Ce texte doit être compris dans le contexte d'une alliance matrimoniale qui vise à assurer la sécurité sociale et économique des femmes de la famille d'un homme sans enfant [9] : Sa-Bastet libère Imeniouy de son statut de serviteur, il en fait son héritier. Imeniouy est ainsi destiné à devenir le chef de famille - et à endosser les responsabilités y afférentes de protection des membres de la maisonnée - après le décès de son ancien maître [10]. Par son engagement à ne pas frapper ni entraver les mouvements de son ancien serviteur, Sa-Bastet garantit la liberté nouvelle qu'il donne à Imeniouy, et renonce ainsi à ses droits de maître sur lui : les liens qui les unissent sont dès lors des liens de parenté, et non plus de subordination. 
Des actes de violence interpersonnelle dans un contexte hiérarchique sont également mentionnés dans les ostraca Mond 237 [11] et ROM 906.19.5 [12], deux copies d'une même lettre, adressée au vizir Khay par le chef des $m \underline{d} 3 y$ Minniouy, sous le règne de Ramsès II [13]. Minniouy se plaint de Nakhtsobek, également chef des $m \underline{d} 3 y$ : il lui reproche d'avoir battu les $m \underline{d} 3 y$ sous son autorité [14]. La violence subie par les hommes de Minniouy s'ancre toutefois dans des méfaits plus variés : I'homme reproche également à son collègue de s'être emparé de ses champs et de les avoir réattribués à d'autres dignitaires. Nakhtsobek a par ailleurs manqué de respect à Minniouy, en lui tenant les propos suivants : « Je suis jeune et tu es vieux »[15], semblant ainsi lui signifier qu'il est temps de laisser son cadet lui succéder. Les comportements de Nakhtsobek, pris dans leur ensemble, constituent en réalité une remise en cause du commandement de Minniouy en tant que chef des $m \underline{d} 3 y$. En rapportant la bastonnade infligée à ses hommes, ce n'est pas la violence en elle-même que dénonce Minniouy, mais cette contestation de son autorité. La nature du document et son destinataire renforcent d'ailleurs la volonté de voir réaffirmée la légitimité de Minniouy comme but premier de sa démarche : il s'agit en effet d'une lettre adressée au vizir, et non d'une plainte formellement adressée à l'administration vizirale [16]. Le vizir intervient ici en tant que supérieur hiérarchique des chefs des $m \underline{d} 3 y$ [17]. Les $m \underline{d} 3 y$ battus sont ses hommes, si quelqu'un est en droit de les battre, c'est Minniouy lui-même, et non un autre chef des $m \underline{d}_{3} y$. Il transparaît ainsi en arrièreplan de ce texte une légitimité de la violence exercée par un supérieur hiérarchique sur ses subordonnés.

La lettre de I'O. Ashmolean Museum 32 [18] présente également un acte de violence interpersonnelle en lien avec une hiérarchie professionnelle. Dans ce texte issu de la documentation de Deir el-Médina, le lien hiérarchique intervient entre l'instigateur de la violence et son responsable. En effet, rapporte I'auteur de la missive, le supérieur Miy a ordonné à son subordonné Qenna d'infliger « dix coups très forts »[19] à un homme non nommé. Il est probable que la victime désignée et l'auteur de la lettre ne soient qu'une seule et même personne. C'est d'ailleurs Qenna lui-même qui a prévenu l'auteur de la missive de l'ordre qu'il a reçu. Il est impossible de dire si la victime de cette violence était ellemême sous l'autorité de Miy. De même, les raisons de cette bastonnade et son éventuelle justification sont inconnues. Il semble cependant que Qenna, I'assaillant, doive attaquer la victime par surprise : Miy lui aurait en effet ordonné de frapper sa victime au moment où il arriverait vers lui [20]. L'agression serait dès lors bel et bien un acte de violence répréhensible. Le fait que l'auteur de la missive rapporte la menace qui pèse sur la victime, et non une infliction réelle de la bastonnade, laisse néanmoins penser que les coups n'ont pas - encore ? - été portés au moment de la rédaction de la lettre. L'histoire ne nous dit pas si c'est en raison de sa position de supérieur hiérarchique que Miy a ordonné à Qenna de commettre cet acte violent. Il est cependant manifeste que la hiérarchie entre les deux hommes devait constituer une pression dont Miy a pu jouer pour pousser Qenna à obtempérer.

Dans la lettre du $P$. Genève D 187 [21], le maire de Thèbes se plaint au scribe de la Tombe Tjary que son serviteur a été « violemment battu » [22], sans doute par un artisan de Deir el-Médina. Tjary semble avoir justifié la bastonnade, en affirmant que les propos de I'artisan « sont vrais » [23] : ce dernier reproche vraisemblablement un méfait au
[11] KiTCHEN 1989, p. 189-190.

[12] GARDINER 1913, p. 16b-k.

[13] Le contexte de découverte de ces deux ostraca est inconnu, mais le destinataire de la missive (le maire de Thèbes) et l'expéditeur (le chef des $m \underline{d} 3 y$ à Thèbes Ouest) rendent son origine thébaine indiscutable. Le document se rapporte cependant à Deir el-Médina, même s'il n'en est pas directement issu : les fonctions de chef des $m \underline{d} 3 y$ sont en lien avec le village des ouvriers de la nécropole thébaine (cf. McDoweLL 1990, p. 63).

[14] $s w$ qnqn $n 3 y=i m \underline{d} 3 y . w$, « Il battait mes $m \underline{d} 3 y$ ». O. Mond 237, x+4 ; O. ROM 906.19.5, 25.

[15] Ntk ỉy ink šri. O. Mond 237, $\mathrm{x}+4-5$; O. ROM 906.19.5, 26.

[16] Voir, par exemple, la plainte du P. BM EA 10055 (= $P$. Salt 124), adressée à un vizir par un artisan de Deir
el-Médina, et qui reprend le formulaire usuel des plaintes. Cf. Allam 1973, p. 281-287.

[17] McDoweLl 1990, p. 54-55. GRAJeTZKI 2010, p. 193, évoque pour sa part des liens de clientélisme entre les habitants de Deir el-Médina et le vizir. Dans le cas précis de la missive de Minniouy, c'est cependant le rôle de supérieur hiérarchique du haut-dignitaire qui semble primer.

[18] Allam 1973, p. 154.

[19] $i w=k$ rdi.t $n=f 10 n s h(. t) d r . w s p 2$, « Tu feras infliger dix coups très forts ». O. Ashm. Mus. 32, 3-4.

[20] $h f t w n n=f h r s p r r=k$, « au moment où il arrivera vers toi ». O. Ashm. Mus. 32, 2-3.

[21] JANSSEN 1991, pl. 57-58.

[22] $q n q n=f m h w r$ '. P. Genève D 187, verso 4.

[23] hriw m3 '(t) $m n_{3 y}=f m d$.wt, «Or, ses paroles sont vraies! ».P. Genève D 187 , recto 5 . 
serviteur, et I'aurait battu pour cette raison. Cette confiance du scribe ne suffit cependant pas au maire de Thèbes : ce dernier écrit en effet que si la punition corporelle était réellement justifiée, alors il battra luimême à nouveau son serviteur ; mais si elle s'avérait injustifiée, le maire fera infliger un châtiment à l'artisan coupable, en présence de Tjary [24]. Le maire de Thèbes remet ainsi en cause la légitimité de l'artisan à châtier son serviteur. En écrivant qu'il infligera lui-même, si nécessaire, une correction à son domestique, le maire semble en réalité insister sur le fait que c'est à lui de faire battre son serviteur, et non à quelqu'un d'autre. Ce texte révèle ainsi deux aspects importants. D'une part, un maître pouvait battre son serviteur, mais ce droit lui était réservé : c'est au maître d'établir si un châtiment est requis. D'autre part, le texte suggère qu'un maître pouvait prendre la défense de son serviteur face à un comportement abusif. Ce dernier aspect peut être mis en lien avec deux relations qui associent le maître et son serviteur : le serviteur appartient probablement à la maisonnée à la tête de laquelle se trouve son maître [25] ; dès lors il est du devoir du maître de protéger le serviteur en tant que membre de sa maisonnée. Par ailleurs, on peut voir dans les liens qui unissent maître et serviteur une relation proche du patronage : la protection du serviteur est assurée en échange de sa loyauté envers son maître.

Un dernier texte documentaire du Nouvel Empire doit être évoqué : une plainte adressée au vizir [26] et datée de la fin de la XIX ${ }^{\text {e }}$ dynastie. Si la violence est bien présente dans ce texte, I'aspect hiérarchique n'est, pour sa part, pas assuré. Le plaignant, Imennakht, dénonce les méfaits de l'artisan-chef Paneb au sein du village de Deir el-Médina, parmi lesquels des agressions physiques, des meurtres et des menaces de meurtre. Plusieurs de ces actes de violence sont dirigés à l'encontre d'artisans. Or, en tant qu'artisan-chef, Paneb dirigeait une partie des artisans [27] ; il était ainsi leur supérieur hiérarchique direct. Est-ce du fait de sa fonction d'artisan-chef que Paneb s'est permis de frapper les artisans sous sa responsabilité ? Il est possible que cette fonction ait fait naître chez I'homme un sentiment d'impunité [28]. Cependant, il doit être souligné que certains comportements violents qu'Imennakht attribue à Paneb ont été commis alors que I'homme n'avait pas encore accédé à la charge d'artisan-chef [29].

Ainsi, dans les textes documentaires du Nouvel Empire qui dénoncent des actes de violence interpersonnelle dans le cadre d'une relation hiérarchique, l'individu en position de supériorité est toujours l'agresseur ou l'instigateur de l'attaque. Lorsqu'un acte de violence perpétré par un supérieur est dénoncé, ce n'est pas l'idée même de la violence d'un supérieur sur son subalterne qui est remise en cause. En réalité, il s'agit de contester la légitimité de la personne qui inflige la bastonnade : le chef des $m \underline{d} 3 y$ de I'O. Mond 237/O. ROM 906.19.5 et le maire de Thèbes du $P$. Genève $\mathrm{D} 187$ dénoncent ainsi tous les deux que des hommes sous leur autorité ont été frappés. Il ne faut pas envisager ces deux textes comme une remise en cause de la violence hiérarchique, mais comme un rappel de l'autorité des plaignants sur les victimes.

C'est en effet la légitimité du supérieur à frapper son subalterne qui ressort en arrière-plan de ces dénonciations. Il en va de même du contrat de mariage entre la nièce et le serviteur du barbier Sa-Bastet : par ce contrat et l'union contractée, Sa-Bastet libère Imeniouy de son statut de serviteur. La promesse de ne pas le frapper ni restreindre ses mouvements suggère qu'un maître était en droit de frapper son serviteur et de ne pas le laisser libre de ses déplacements.

La littérature du Nouvel Empire est relativement pauvre en témoignages de violence hiérarchique : un seul texte littéraire en fait état, et il s'agit alors d'une hiérarchie sociale. L'Enseignement d'Aménémopé [30] évoque en effet l'exercice de la violence à l'encontre d'une personne en situation d'infériorité sociale. Dans une maxime de cette sagesse datée de la toute fin du Nouvel Empire [31], il est conseillé
[24] $i w=i$ rdi.t $p t r=k$ p $n t y$ iw $=i$ ir. $[t w=f n=f . .$.$] , « je$ m'arrangerai pour que tu vois ce que je ferai en sorte qu'on lui inflige [à lui...] ». P. Genève D 187, verso 4.

[25] Sur la composition des maisonnées, cf. Donnat Beauquier 2014, p. 174 et Frood 2010, p. 473.

[26] P. BM EA 10055 (= P. Salt 124), cf. AlLAm 1973, p. 281-287.

[27] Il n'y avait jamais plus de deux artisans-chefs à la fois à Deir el-Médina. Chacun dirigeait une partie de l'équipe des artisans (la droite ou la gauche) ; cf. Cerný 2001, p. $121-123$.

[28] Un tel sentiment d'impunité ne serait pas nouveau pour Paneb : selon la plainte d'Imennakht, l'artisan-chef aurait réussi à faire démissionner un vizir en place. $C f$. P. BM EA 10055, recto II 17-18.

[29] Dans $P$. BM EA 10055, recto II 14-18, Paneb attaque Neferhotep et menace de le tuer : ce dernier est donc encore vivant. Or, Paneb a justement hérité de la charge d'artisan-chef de Neferhotep à sa mort : il ne pouvait donc pas détenir une telle fonction lors de cette agression. Cf. Davies 1999, p. 35-36.

[30] P. BM EA 10474, IV 4-6, cf. LAISNEY 2007, p. 56.

[31] Le texte doit être situé à la fin de $X X$ ou au début de la XXI dynastie. Cf. Vernus 2010, p. 389-390. 


\begin{tabular}{|c|c|c|c|c|c|c|c|}
\hline & P. ASSOC. 1 & $\begin{array}{l}\text { P. VOGL. INV. } \\
\text { DEM. } 77\end{array}$ & P. ASSOC. 3 & P. ASSOC. 5 & P. ASSOC. 6 & P. ASSOC. 7 & P. ASSOC. 8 \\
\hline & 223 av. J.-C. & 178 av. J.-C. & 157 av. J.-C. & 147 av. J.-C. & 145 av. J.-C. & 137 av. J.-C. & 137 av. J.-C. \\
\hline Battre un autre membre & & 400 & 600 & 1000 & 500 & $1 ? ? ?$ & 500 \\
\hline Battre le supérieur/président & 8 & 400 & ??? & 1200 & 650 & & 1000 \\
\hline Réciproque & 10 & $400(?)$ & 800 & 1500 & 850 & & 1500 \\
\hline Battre le second & & $?$ & ??? & 800 & 600 & & 1000 \\
\hline Réciproque & & 400 & $? 50$ & 900 & 750 & & 1500 \\
\hline Battre un prêtre ordinaire & & & 800 & 1000 & 800 & & \\
\hline Réciproque & & & 900 & 1500 & 1000 & & \\
\hline Battre le lésonis & & $?$ & & & & & \\
\hline $\begin{array}{l}\text { Battre celui qui répartit } \\
\text { les fonctions }\end{array}$ & & & & & & & 1000 \\
\hline Réciproque & & & & & & & 1500 \\
\hline Frapper un novice & & & & & & & 100 \\
\hline
\end{tabular}

Figure 2 : actes de violence prohibés et montants des amendes (convertis en kité) dans les règlements d'association démotiques.

au lecteur de ne pas brutaliser un faible [32]. Elle $s^{\prime}$ ancre dans un passage plus global, qui déconseille toute action hostile envers une personne en situation d'infériorité sociale ou physique : ne pas voler un misérable $\left(h w r^{\prime}{ }^{i} 3 d\right)$, ne pas brutaliser un faible $(n ` s s 3 w-')$ et ne pas tendre la main pour approcher

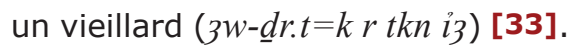

L'objectif de cette maxime n'est donc pas, en soi, de déconseiller la violence : il s'agit plutôt d'inviter toute personne en situation de supériorité - fût-elle sociale, économique ou physique - à ne pas persécuter des individus en position de vulnérabilité.

\section{PRÉVENTION ET ÉLOGE DE LA VIOLENCE HIÉRARCHIQUE À L'ÉPOQUE HELLÉNISTIQUE}

Rares sont les textes documentaires d'époque hellénistique qui mentionnent une violence interpersonnelle en lien avec une relation hiérarchique. En réalité, dans les documents de la pratique quotidienne, seule une catégorie de textes est concernée : les règlements d'associations démotiques.

Les règlements d'associations énumèrent les règles que les membres d'une association religieuse et professionnelle, le plus souvent composée de prêtres, devaient respecter [34]. Sept de ces règlements démotiques, datés entre 223 et 137 av. J.-C., mentionnent des actes de violence parmi les comportements prohibés au sein des associations concernées (Fig. 2).

L'élément hiérarchique intervient, dans ces documents, non pas dans la description des agressions prohibées, mais dans les amendes qui sanctionnent quiconque exercerait cette violence. En effet, les peines pécuniaires ne varient pas en fonction de l'acte violent commis, mais selon le statut de la victime et de son agresseur au sein de l'association. Il est ainsi frappant de remarquer que I'amende d'un individu au statut important est plus élevée $s^{\prime}$ il agresse un membre au statut inférieur, que ne l'est celle d'un membre ordinaire qui s'en prendrait physiquement à ce personnage important. L'aspect hiérarchique dans le montant des amendes s'exprime donc systématiquement [35] en faveur des membres en position d'infériorité.

Dans les règlements d'associations d'Égypte hellénistique, la hiérarchie est en lien avec une hiérarchie professionnelle : les membres les plus importants d'une association étaient des individus hauts placés dans la hiérarchie ecclésiastique [36]. Les raisons

[32] $S_{3} w t w[\ldots] r n \stackrel{s}{s} s_{3} w$ -

[33] Le terme $t k n$, dans cette occurrence spécifique, est la plupart du temps interprété comme un geste violent (voir notamment LAISNEY 2007, p. 55 et VeRNUS 2010, p. 400). Si l'expression « tendre la main » qui lui est associée invite en effet à lui attribuer un tel sens, en réalité rien ne permet d'assurer que $t k n$ exprime une violence physique. Je lui préfère donc le terme plus neutre « approcher » (cf. Wb. V, 333.10-334.20).

[34] Pour l'ensemble des règlements d'associations rédigés en démotique, cf. CénIVAL 1972 et BRESCIANI 1994, p. 46-67.

[35] À l'exception du P. Vogl. Inv. Dem. 77 ; ce texte est cependant lacunaire, toutes les amendes ne sont donc pas connues (cf. BRESCIANI 1994).

[36] CÉnIVAL 1972. Sur l'aisance financière des membres d'associations, cf. Monson 2007, p. 237. 
de ce déséquilibre des amendes en faveur des membres en situation d'infériorité - qui n'est pas propre aux actes de violence - ne sont pas clairement établies. Cette caractéristique pourrait avoir eu pour fonction d'éviter les abus de pouvoir de la part de personnages aux positions importantes au sein de I'association. D'autres possibilités sont cependant envisageables, comme celle de l'adaptation du montant des sanctions aux moyens financiers des membres, ou de la volonté de donner un exemple de comportement convenable. Andrew Monson [37] s'est étonné du montant très élevé de certaines amendes dans les règlements d'associations démotiques. Le chercheur considère ainsi que les chiffres énoncés pourraient être fictifs : les peines pécuniaires auraient eu comme seul but de décourager les comportements immoraux. La dissuasion est clairement un objectif essentiel de ces sanctions. Je pense néanmoins que leur montant était réel et non fictif : un membre qui aurait contrevenu à ces règles aurait bel et bien dû s'acquitter de I'amende énoncée. En effet, quel serait le réel impact dissuasif d'une sanction jamais véritablement infligée [38] ?

Les règlements d'associations sont ainsi les seuls textes démotiques de la pratique quotidienne de la dynastie lagide à mentionner une violence interpersonnelle hiérarchique. La littérature démotique n'est guère plus prolixe : un texte littéraire d'époque hellénistique évoque une violence dans le cadre d'une hiérarchie, et il ne s'agit alors pas d'une violence interpersonnelle.

En effet, I'Enseignement d'Ankhchéchonky [39], texte sapiential composé entre le $v^{\mathrm{e}}$ et le $\mathrm{I}^{\mathrm{er}}$ siècle av. J.-C., loin de déconseiller I'usage de la violence - comme c'était le cas dans la sagesse de la fin du Nouvel Empire [40] —, vante au contraire les vertus de la bastonnade infligée à un serviteur : « Un serviteur qui n'est pas battu, la malédiction est grande dans son cœur »[41]. Ce passage est ainsi centré sur la victime - le serviteur - et non sur celui qui inflige la violence. La violence d'un maître sur son domestique est ici légitimée : il s'agit de corriger

[37] Monson 2006, p. 230-233.

[38] Il est néanmoins possible qu'à partir de ces montants importants, les amendes aient ensuite été négociées individuellement. L'estimation individuelle des contraventions dans les associations est attestée plus tardivement, notamment par le règlement grec d'association du $P$. Mich. $V$, 243, daté du règne de Tibère (cf. HusSELMAN, BOAK \& EDGERTON 1944, p. 90).

[39] P. BM EA 10508 C, cf. GLANVILLE 1955.

[40] Cf. supra. le domestique pour le rendre meilleur. Faute de bastonnade, la « malédiction » régnerait dans son cœur. Cette justification de la violence exercée sur un serviteur signifie qu'elle n'est pas perçue comme un acte de violence interpersonnelle par l'auteur du texte, mais comme un geste nécessaire pour assurer la loyauté du serviteur.

Une autre maxime de I'Enseignement d'Ankhchéchonqy se concentre pour sa part sur la violence que peut infliger un supérieur, fut-il bienveillant : «Un supérieur bienveillant, il tue pour plaire » [42]. À mon sens, cette maxime signifie que la violence du maître ou du supérieur peut frapper sans prévenir, pour son simple plaisir. Cette sagesse présente ainsi une vision légitimée de la violence d'un maître sur son serviteur, d'un supérieur sur son subalterne, sans que ce dernier puisse s'y soustraire. Il est intéressant de noter, dans ce passage, que la violence n'est pas justifiée par une quelconque nécessité, mais relève de la seule impulsion de l'individu en position de supériorité.

Enfin, il convient de mentionner une deuxième œuvre littéraire d'époque hellénistique : la sagesse du $P$. Insinger [43], datée du $I^{\text {er }}$ siècle av. J.-C. En effet, si ce texte n'évoque pas directement une violence hiérarchique, son auteur présente néanmoins à plusieurs reprises les vertus du bâton ( $̌ s b t)$ comme instrument de la bastonnade : « C'est pour instruire le perturbateur que Thot a laissé (le) bâton sur la terre »[44]; «Bâton et honte sont la protection de son fils contre le scélérat » [45]; « La loi et la justice disparaissent dans un village quand il n'y a pas de bâton » [46].

L'auteur de ce texte met ainsi en avant une violence justifiée, de son point de vue, car elle vient sanctionner un acte de déviance, qui ne constitue donc pas une violence interpersonnelle : le bâton participe au maintien de l'ordre et à l'éviction du chaos. Or, si la violence du bâton s'exerce sur le « perturbateur » et le «scélérat », elle s'exerce également sur le serviteur lorsque son maître le bat pour le corriger. Le bâton est l'outil du maintien de l'ordre dans la société. Maintenir l'ordre, ce n'est

[41] b3k iw bw-ir=w mhy.t=f n3-'3 shwr $m$ ḩ3t.t=f ; P. BM EA 10508 C VII 18.

[42] hry $m r-r m \underline{t} i . i r=f \underline{h} t b r h r$; P. BM EA 10508 C XII 19. [43] VOLTEN 1941.

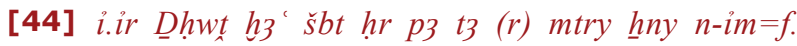
$P$. Insinger, IX 6 .

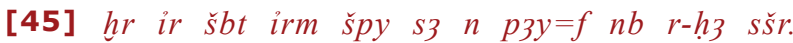
$P$. Insinger, IX 11.

[46] hr lg hp md(.t)-m3'.t hhn tmy î-mn šbt. P. Insinger, XIV 16. 
pas seulement mettre à l'écart les perturbateurs et autres scélérats, c'est aussi assurer le maintien de la société, et par là même des normes et des hiérarchies qui la structurent.

Ces sagesses démotiques louent les mérites d'une violence hiérarchique et des vertus du bâton : elles tranchent ainsi avec la sagesse du Nouvel Empire, évoquée plus haut, qui déconseillait d'agresser une personne en situation d'infériorité sociale, économique ou physique [47].

Ces deux textes sapientiaux doivent cependant être observés à la lumière de la société des villages du premier millénaire av. J.-C. : il s'agit d'une société fortement hiérarchisée, dominée par ses notables [48]. Le contrôle social de I'individu par la communauté tient une place importante [49] : il s'exprime dans les sagesses à travers l'éloge du bâton et de la nécessité de battre un serviteur.

\section{CONCLUSION}

De nombreuses convergences ressortent de I'étude des documentations néo-égyptienne et démotique. La violence d'un supérieur sur son subalterne ou d'un maître sur son serviteur semble être, dans I'ensemble, socialement acceptée autant dans les textes du Nouvel Empire que dans ceux d'époque hellénistique. Cette violence doit toutefois être utilisée à bon escient et avec mesure : ainsi, l'Enseignement d'Aménémopé invite son lecteur à ne pas s'acharner sur plus faible que soi. Les règlements d'associations tentent quant à eux de limiter les abus en punissant plus durement la violence exercée par des membres importants de I'association.

L'image du noble qui ne doit pas faire mauvais usage de son pouvoir sur le vulnérable ou le pauvre n'est pas propre à l'Égypte du Nouvel Empire et de I'époque hellénistique : elle est déjà présente dans des textes de I'Ancien et du Moyen Empire. Ces documents soulignent en effet le principe de « réciprocité sociale » et critiquent un mauvais emploi

[47] Noter cependant, pour le Nouvel Empire, le cas particulier de la violence exercée par le maître d'école sur son élève dans le cadre de l'apprentissage quotidien, qui semble être socialement acceptée. Voir par exemple le $P$. Sallier I, 7,7-8,2, ou le $P$. Anastasi III, recto 3,9-4,4. Cf. Vernus 2010, p. 478-479.

[48] Agut-Labordère 2011, p. 110-111.

[49] Agut-Labordère 2011, p. 114.

[50] DONNAT (à paraître). des richesses-špssw, qui ne s'inscrirait pas « dans un projet économique et social collectif » [50].

Lorsque la violence hiérarchique est dénoncée, ce n'est pas l'institution en elle-même qui est remise en cause, mais l'autorité de la personne qui exerce la violence : on reproche ainsi à un chef des $m \underline{d} 3 y$ et à un artisan d'avoir battu des hommes sur lesquels ils n'avaient pas autorité. De telles dénonciations, uniquement relevées dans le corpus du Nouvel Empire, ne sont pas le fait des victimes de la bastonnade, mais de leurs maîtres ou supérieurs légitimes, dans le but de réaffirmer leur autorité.

Si les documents du Nouvel Empire et d'époque hellénistique présentent de nombreux points de convergence sur la violence interpersonnelle en contexte hiérarchique, une différence doit cependant être soulignée. Elle réside dans la perception de ce phénomène apportée par chacune des grandes catégories de textes. En effet, au Nouvel Empire, seuls les textes documentaires laissent transparaître la légitimité d'un supérieur à battre son subalterne, tandis que I'Enseignement d'Aménémopé invite à agir avec mesure face à un individu en position d'infériorité. Au contraire, à l'époque hellénistique, ce sont les règlements d'associations qui tentent d'éviter les excès des individus importants, tandis que la littérature justifie pour sa part l'infliction d'un châtiment violent à un serviteur, affirmant ainsi la légitimité d'une telle violence. Cette différence entre les textes littéraires néo-égyptiens et démotiques trouve une explication partielle dans une évolution de la figure du scribe : au premier millénaire, la « classe moyenne provinciale » [51] rédige des textes documentaires et littéraires en démotique, alors qu'elle n'écrivait que très peu au second millénaire av. J.-C. [52]. Les sagesses des scribes d'époque hellénistique concernent ainsi le fonctionnement de la société locale et s'écartent de la vie des grandes institutions que décrivaient les sagesses du Nouvel Empire [53]. Elles nous renseignent sur le village égyptien et la structuration de sa hiérarchie sociale gouvernée par les notables [54], et non une élite
[51] Agut-Labordère 2011, p. 108.

[52] Ibid., p. 108.

[53] Ibid., p. 108-109.

[54] Ibid., p. 110-111. L'impact des corpus de textes mis au jour ne doit pas être négligé : en dehors de Deir el-Médina, les villages du Nouvel Empire sont très peu mentionnés par la documentation. C'est la vie de l'élite de la société dans la capitale égyptienne, à travers les biographies des hauts dignitaires, qui est la plus éclairée pour cette époque. 
qui se montre par sa richesse mais aussi par sa générosité et sa protection des plus faibles comme au Nouvel Empire. Le contraste entre les littératures du Nouvel Empire et de l'époque hellénistique n'est donc pas révélateur d'une rupture dans les pratiques ou les mentalités, mais plutôt des milieux distincts dont sont issus les auteurs de ces textes, leur procurant une perception différente de l'exercice de la violence envers un individu en position d'infériorité.

Ce contraste s'explique également par le contexte des violences hiérarchiques. En effet, dans la sagesse néo-égyptienne de l'Enseignement d'Aménémopé, la hiérarchie sociale s'exprime du point de vue du rang, de la position dans la société égyptienne, d'une vulnérabilité économique et physique. La notion d'infériorité n'est ainsi pas liée à une subordination professionnelle ou à un assujettissement. Au contraire, les sagesses démotiques du $P$. Insinger et de I'Enseignement d'Ankhchéchonqy décrivent une relation entre maître et serviteur, qui rejoint celle des textes documentaires du Nouvel Empire : le serviteur est subordonné à son maître et appartient à sa maisonnée.

Le maître est avant tout en effet le chef de famille. Il possède une forme de patria potestas sur sa maisonnée, y compris ses serviteurs. Il ne faut pas envisager la patria potestas dans son sens le plus fort, comme celle du pater familias de la Rome antique [55], mais, dans le contexte présent, comme la possibilité pour le chef de famille d'infliger une correction corporelle à sa femme, ses enfants ou ses serviteurs [56]. L'analyse des textes indique néanmoins que cette correction doit être justifiée et ne pas être abusive.
Les relations de pouvoir qui structurent la société égyptienne apportent une protection au faible et à I'opprimé face aux abus des individus en position de supériorité hiérarchique, et influencent ainsi la gestion de la violence interpersonnelle. Ces caractéristiques communes aux textes du Nouvel Empire et d'époque hellénistique indiquent une absence de rupture nette dans le traitement social de la violence en contexte hiérarchique entre les deux périodes. Elles s'ancrent en réalité dans une continuité des phénomènes sociaux, peu touchés par les fluctuations de la vie politique.

Il ressort de l'étude des textes égyptiens du Nouvel Empire et d'époque hellénistique que la violence hiérarchique est perçue comme légitime aux deux périodes étudiées. Il ne s'agit donc pas d'une violence interpersonnelle. Pourtant, cette violence devient interpersonnelle dès lors qu'elle est exercée par un individu qui n'a pas autorité sur la personne qu'il frappe, ou que son utilisation est jugée abusive ou injustifiée. La perception de cette violence par sa victime ou son dénonciateur est en effet ce qui permet à l'observateur moderne d'identifier l'usage d'une force physique comme une violence légitime ou interpersonnelle, et de tenter ainsi de se rapprocher du point de vue interne à la société égyptienne.

[55] Sur l'exercice de la patria potestas du chef de famille dans l'Égypte romaine, cf. HUEBNER 2013, p. 66, 153 et 184 . Sur la patria potestas dans le droit romain, cf. Dixon 1992, p.3-5.

[56] Une maxime de la sagesse démotique du $P$. Louvre 2414 enjoint ainsi son lecteur à battre sa femme et empoigner fermement son fils, si I'un ou l'autre l'insultait. Cf. HuE-ARCÉ (à paraître).

\section{BIBLIOGRAPHIE}

Agut-Larbordère, Damien, 2011, «Les "petites citadelles". La sociabilité du tmy "ville", "village" à travers les sagesses démotiques », dans Gilles Gorre \& Perrine Kossmann (éd.), Espaces et territoires de l'Égypte gréco-romaine. Actes des journées d'étude, 23 juin 2007 et 21 juin 2008. Cahiers de l'atelier Aigyptos 1, Genève (Hautes Études du Monde GrécoRomain), p. 107-122.

Allam, Schafik, 1973, Hieratische Ostraka und Papyri aus der Ramessidenzeit, Tübingen (Urkunden zum Rechtsleben im Alten Ägypten 1).

Bresciani, Edda, 1994, « Nuovi statuti demotici di "Confraternite" dalla necropoli dei Crocodilli a Tebtynis (P. Vogl. demot. Inv. 77 et Inv. 78) », Egitto e Vicino Oriente 17, p. 46-67.

CénIVAL, Françoise de, 1972, Les Associations religieuses en Égypte: d'après les documents démotiques, Le Caire (Bibliothèque d'Étude 46).

Černý, Jaroslav, 2001, A Community of workmen at Thebes in the Ramesside Period, $2^{\mathrm{e}}$ éd. (1re éd. 1973), Le Caire (Bibliothèque d'Étude 50). 
Davies, Benedict G., 1999, Who's who at Deir el-Medina. A Prosographic study of the royal workmen's community, Leiden (Egyptologische Uitgaven 13).

Dieleman, Jacco, 1998, « Fear of women? Representations of women in Demotic wisdom texts », Studien für Altägyptischen Kultur 25, p. 7-46.

Dixon, Suzanne, 1992, The Roman family, Baltimore.

Donnat, Sylvie, à paraître, «Du luxe aux richesses-špssw. À propos de la scène du "petit-lever" de Ptahhotep (Égypte, vers 2400-2300 av. J.-C.) », dans Stavros Lazaris (éd.), Donner à voir : la mise en scène du luxe dans les cultures visuelles de l'Antiquité, Turnhout.

Donnat Beauquier, Sylvie, 2014, Écrire à ses morts. Enquête sur un usage rituel de l'écrit dans l'Égypte ancienne, Grenoble (Horos).

EYre, Christopher, 1992, «The Adoption papyrus in context », The Journal of Egyptian Archaeology 78, p. 207-221.

Frood, Elizabeth, 2010, «Social structure and daily life: Pharaonic », dans Alan B. Lloyd (éd.), A Companion to ancient Egypt, Malden - Oxford - Chichester, p. 469-490.

GaRdiner, Alan H., 1913, Theban ostraca edited from the originals, now mainly in the Royal Ontario Museum of Archaeology, Toronto, and the Bodleian Library, Oxford, Toronto - London - Oxford (University of Toronto Studies).

Glanville, Stephen R. K., 1955, Catalogue of Demotic papyri in the British Museum II. The instructions of 'Onchsheshonqy, London (British Museum Papyrus 10508).

Grajetzki, Wolfram, 2010, «Class and Society. Position and Possessions », dans Willeke Wendrich (éd.), Egyptian archaeology, Malden - Oxford - Chichester (Blackwell Studies in Global Archaeology).

Huf-Arć́, Christine, 2015, La violence interpersonnelle dans la documentation égyptienne au Nouvel Empire et aux époques grecque et romaine ( $X V I^{e}-X^{e} S$. av. n. ère / IVe $s . a v$. - IVe $s$. de $n$. ère), Thèse de doctorat, Université de Strasbourg. Hue-Arcé, Christine, à paraître, «Violence against women in Graeco-Roman Egypt: the contribution of Demotic documents », dans Uroš Matić \& Bo Jensen (éd.), Archaeologies of gender and violence, Oxford, p. 133-150.

Huebner, Sabine, 2013, The family in Roman Egypt. A comparative approach to intergenerational solidarity and conflict, Cambridge.

Husselman, Elinor M., Boak, Arthur E. R. \& Edgerton, William F. (éd.), 1944, Papyri from Tebtunis. Part II, Ann Arbor, (University of Michigan Studies. Humanistic Series 29; Michigan Papyri 5).

JANSSEN, Jac. J., 1991, Late Ramesside letters and communications, London (Hieratic Papyri in the British Museum 6). Johnson, Janet H., 1996, « The legal status of women in ancient Egypt », dans Anne K. Capel \& Glenn E. Markoe (éd.), Mistress of the House, Mistress of the Heaven: women in ancient Egypt, New York, p. 175-186.

KITCHEN, Kenneth A., 1989, Ramesside inscriptions. Historical and bibliographical. Volume VII, Oxford.

LAISNEY, Vincent P.-M., 2007, L'Enseignement d'Aménémopé, Roma (Studia Pohl (Series Maior) 19).

LinAGE, Joseph de, 1939, «L'acte d'établissement et le contrat de mariage d'un esclave sous Thoutmosis III », Bulletin de l'Institut Français d'Archéologie Orientale 38, p. 217-234.

McDowelL, Andrea G., 1990, Jurisdiction in the workmen's community of Deir el-Medîna, Leiden (Egyptologische Uitgaven 5).

Monson, Andrew, 2006, «The ethics and economics of Ptolemaic religious associations », Ancient Society 36, p. 221-238.

Monson, Andrew, 2007, « Religious associations and temples in Ptolemaic Tebtunis », dans Jaakko Frösén et al. (éd.), Proceedings of the 24th international congress of papyrology: Helsinki, 1-7 August, 2004, Helsinki (Commentationes Humanarum Litterarum 122), p. 769-779.

PIKE, Kenneth L., 1967, Language in relation to an unified theory of the structure of human behavior, Paris - La Hague. RIESS, Werner, 2012, Performing interpersonal violence. Court, curse, and comedy in fourth-century BCE Athens, Berlin - Boston (MythosEikonPoiesis 4).

Toivari ViItala, Jaana, 2001, Women at Deir el-Medina: a study of the status and roles of the female inhabitants in the workmen's community during the Ramesside period, Leiden (Egyptologische Uitgaven 15).

Vernus, Pascal, 2010, Sagesses de l'Égypte pharaonique, $2^{\mathrm{e}}$ éd. (1 $1^{\text {re }}$ éd. 2001), Arles.

Volten, Aksel, 1941, Das demotische Weisheitsbuch, København (Analecta Aegyptiaca 2).

WARD, William A., 1994, « Foreigners living in the Village », dans Leonard H. Lesko (éd.), Pharaoh's workers. The villagers of Deir el Medina, London, p. 61-86. 